\title{
Prime ends in the mapping theory on the Riemann surfaces
}

\author{
Vladimir Ryazanov, Sergei Volkov \\ (Presented by V. Ya. Gutlyanskii)
}

\begin{abstract}
It is proved criteria for continuous and homeomorphic extension to the boundary of mappings with finite distortion between domains on the Riemann surfaces by prime ends of Caratheodory.
\end{abstract}

2010 MSC. Primary 31A05, 31A20, 31A25, 31B25, 35Q15; Secondary 30E25, 31C05, 34M50, 35F45.

Key words and phrases. Prime ends, Riemann sufaces, mappings of finite distortion, boundary behavior, Sobolev classes.

\section{Introduction}

The theory of the boundary behavior in the prime ends for the mappings with finite distortion has been developed in [12] for the plane domains and in [15] for the spatial domains. The pointwise boundary behavior of the mappings with finite distortion in regular domains on Riemann surfaces was recently studied by us in [30] and [31]. Moreover, the problem was investigated in regular domains on the Riemann manifolds for $n \geq 3$ as well as in metric spaces, see e.g. [1] and [34]. It is necessary to mention also that the theory of the boundary behavior of Sobolev's mappings has significant applications to the boundary value problems for the Beltrami equations and for analogs of the Laplace equation in anisotropic and inhomogeneous media, see e.g. $[3,8,10,11,13,14,20,23,26]$ and relevant references therein.

For basic definitions and notations, discussions and historic comments in the mapping theory on the Riemann surfaces, see our previous papers [29-32].

Received 26.03.2017 


\section{Definition of the prime ends and preliminary remarks}

We act similarly to Caratheodory [5] under the definition of the prime ends of domains on a Riemann surface $\mathbb{S}$, see Chapter 9 in [6]. First of all, recall that a continuous mapping $\sigma: \mathbb{I} \rightarrow \mathbb{S}, \mathbb{I}=(0,1)$, is called a Jordan arc in $\mathbb{S}$ if $\sigma\left(t_{1}\right) \neq \sigma\left(t_{2}\right)$ for $t_{1} \neq t_{2}$. We also use the notations $\sigma, \bar{\sigma}$ and $\partial \sigma$ for $\sigma(\mathbb{I}), \overline{\sigma(\mathbb{I})}$ and $\overline{\sigma(\mathbb{I})} \backslash \sigma(\mathbb{I})$, correspondingly. A Jordan arc $\sigma$ in a domain $D \subset \mathbb{S}$ is called a cross-cut of the domain $D$ if $\sigma$ splits $D$, i.e. $D \backslash \sigma$ has more than one (connected) component, $\partial \sigma \subseteq \partial D$ and $\bar{\sigma}$ is a compact set in $\mathbb{S}$.

A sequence $\sigma_{1}, \ldots, \sigma_{m}, \ldots$ of cross-cuts of $D$ is called a chain in $D$ if:

(i) $\overline{\sigma_{i}} \cap \overline{\sigma_{j}}=\varnothing$ for every $i \neq j, i, j=1,2, \ldots$;

(ii) $\sigma_{m}$ splits $D$ into 2 domains one of which contains $\sigma_{m+1}$ and another one $\sigma_{m-1}$ for every $m>1$;

(iii) $\delta\left(\sigma_{m}\right) \rightarrow 0$ as $m \rightarrow \infty$.

Here $\delta(E)=\sup _{p_{1}, p_{2} \in \mathbb{S}} \delta\left(p_{1}, p_{2}\right)$ denotes the diameter of a set $E$ in $\mathbb{S}$ with respect to an arbitrary metric $\delta$ in $\mathbb{S}$ agreed with its topology, see [29][31].

Correspondingly to the definition, a chain of cross-cuts $\sigma_{m}$ generates a sequence of domains $d_{m} \subset D$ such that $d_{1} \supset d_{2} \supset \ldots \supset d_{m} \supset \ldots$ and $D \cap \partial d_{m}=\sigma_{m}$. Two chains of cross-cuts $\left\{\sigma_{m}\right\}$ and $\left\{\sigma_{k}^{\prime}\right\}$ are called equivalent if, for every $m=1,2, \ldots$, the domain $d_{m}$ contains all domains $d_{k}^{\prime}$ except a finite number and, for every $k=1,2, \ldots$, the domain $d_{k}^{\prime}$ contains all domains $d_{m}$ except a finite number, too. A prime end $P$ of the domain $D$ is an equivalence class of chains of cross-cuts of $D$. Later on, $E_{D}$ denote the collection of all prime ends of a domain $D$ and $\bar{D}_{P}=D \cup E_{D}$ is its completion by prime ends.

Next, we say that a sequence of points $p_{l} \in D$ is convergent to a prime end $P$ of $D$ if, for a chain of cross-cuts $\left\{\sigma_{m}\right\}$ in $P$, for every $m=1,2, \ldots$, the domain $d_{m}$ contains all points $p_{l}$ except their finite collection. Further, we say that a sequence of prime ends $P_{l}$ converge to a prime end $P$ if, for a chain of cross-cuts $\left\{\sigma_{m}\right\}$ in $P$, for every $m=1,2, \ldots$, the domain $d_{m}$ contains chains of cross-cuts $\left\{\sigma_{k}^{\prime}\right\}$ in all prime ends $P_{l}$ except their finite collection.

Now, let $D$ be a domain in the compactification $\overline{\mathbb{S}}$ of a Riemann surface $\mathbb{S}$ by Kerekjarto-Stoilow, see a discussion in [29]- [31]. Denote by $E_{D}$ the union of $D$ and all its prime ends. Open neighborhoods of points in $D$ is induced by the topology of $\overline{\mathbb{S}}$. A basis of neighborhoods of a prime end $P$ of $D$ can be defined in the following way. Let $d$ be an arbitrary domain from a chain in $P$. Denote by $d^{*}$ the union of $d$ and all 
prime ends of $D$ having some chains in $d$. Just all such $d^{*}$ form a basis of open neighborhoods of the prime end $P$. The corresponding topology on $\bar{D}_{P}$ is called the topology of prime ends.

Let $P$ be a prime end of $D$ on a Riemann surface $\mathbb{S},\left\{\sigma_{m}\right\}$ and $\left\{\sigma_{m}^{\prime}\right\}$ be two chains in $P, d_{m}$ and $d_{m}^{\prime}$ be domains corresponding to $\sigma_{m}$ and $\sigma_{m}^{\prime}$. Then

$$
\bigcap_{m=1}^{\infty} \overline{d_{m}} \subseteq \bigcap_{m=1}^{\infty} \overline{d_{m}^{\prime}} \subset \bigcap_{m=1}^{\infty} \overline{d_{m}},
$$

and, thus,

$$
\bigcap_{m=1}^{\infty} \overline{d_{m}}=\bigcap_{m=1}^{\infty} \overline{d_{m}^{\prime}},
$$

i.e. the set named by a body of the prime end $P$

$$
I(P):=\bigcap_{m=1}^{\infty} \overline{d_{m}}
$$

depends only on $P$ but not on a choice of a chain of cross-cuts $\left\{\sigma_{m}\right\}$ in $P$.

It is necessary to note also that, for any chain $\left\{\sigma_{m}\right\}$ in the prime end $P$,

$$
\Omega:=\bigcap_{m=1}^{\infty} d_{m}=\varnothing .
$$

Indeed, every point $p$ in $\Omega$ belongs to $D$. Moreover, some open neighborhood of $p$ in $D$ should belong to $\Omega$. In the contrary case each neighborhood of $p$ should have a point in some $\sigma_{m}$. However, in view of condition (iii) then $p \in \partial D$ that should contradict the inclusion $p \in D$. Thus, $\Omega$ is an open set and if $\Omega$ would be not empty, then the connectedness of $D$ would be broken because $D=\Omega \cup \Omega^{*}$ with the open set $\Omega^{*}:=D \backslash I(P)$.

In view of conditions (i) and (ii), we have by (2.2) that

$$
I(P)=\bigcap_{m=1}^{\infty}\left(\partial d_{m} \cap \partial D\right)=\partial D \cap \bigcap_{m=1}^{\infty} \partial d_{m} .
$$

Thus, we obtain the following statement.

Proposition 2.1. For each prime end $P$ of a domain $D$ on a Riemann surface,

$$
I(P) \subseteq \partial D .
$$


Remark 2.1. If $\partial D$ is a compact set in $\mathbb{S}$, then $I(P)$ is a continuum, i.e. it is a connected compact set, see e.g. I(9.12) in [37], see also I.9.3 in [4], and $I(P)$ belongs to only one (connected) component $\Gamma$ of $\partial D$. In the case, we say that the component $\Gamma$ is associated with the prime end $P$.

Moreover, in the case of a compact boundary of $D$, every prime end of $D$ contains a convergent chain $\left\{\sigma_{m}\right\}$, i.e., that is contracted to a point $p_{0} \in \partial D$. Furthermore, each prime end $P$ contains a spherical chain $\left\{\sigma_{m}\right\}$ lying on circles $S\left(p_{0}, r_{m}\right)=\left\{p \in \mathbb{S}: \delta\left(p, p_{0}\right)=r_{m}\right\}$ with $p_{0} \in \partial D$ and $r_{m} \rightarrow 0$ as $m \rightarrow \infty$. The proof is perfectly similar to Lemma 1 in [15] after the replacement of metrics, see also Theorem 7.1 in [22], and hence we omit it. Note by the way that condition (iii) does not depend on the choice of the metric $\delta$ agreed with the topology of $\mathbb{S}$ because $\partial D$ has a compact neighborhood.

\section{The main lemma}

Lemma 3.1. Let $D$ be a domain in a Riemann surface $\mathbb{S}$ and let $\Gamma$ be a compact isolated component of $\partial D$ in $\mathbb{S}$ that is not degenerated to a point. Then $\Gamma$ has a neighborhood $U$ with a conformal mapping $h$ of $U^{*}:=U \cap D$ onto a ring $R=\{z \in \mathbb{C}: 0<r<|z|<1\}$ where one may assume that $\gamma:=\partial U^{*} \cap D$ is a closed Jordan curve and

$$
C(\gamma, h)=\{z \in \mathbb{C}:|z|=1\}, C(\Gamma, h)=\{z \in \mathbb{C}:|z|=r\} .
$$

Furthermore, the map $h$ can be extended to a homeomorphism of $E_{U^{*}}$ onto $\bar{R}$.

Here we use the notation of the cluster set of the mapping $h$ for $B \subseteq \partial D$,

$$
C(B, h):=\left\{z \in \mathbb{C}: z=\lim _{k \rightarrow \infty} h\left(p_{k}\right), p_{k} \rightarrow p \in B, p_{k} \in D\right\}
$$

Note that the first statement is obvious in the case of isolated boundary points of $\partial D$ with $r=0$ and the punctured unit disk $R=\mathbb{D}_{0}:=\{z \in \mathbb{C}$ : $0<|z|<1\}$.

Proof. By the Kerekjarto-Stoilow representation of $\mathbb{S}$, see a discussion in [29]- [31], $\Gamma$ has an open neighborhood $V$ in $\mathbb{S}$ of a finite genus. Without loss of generality, we may assume that $V$ is connected and does not intersect $\partial D \backslash \Gamma$ because $\Gamma$ is an isolated component of $\partial D$. Thus, $V \cap D$ is a Riemann surface of finite genus with an isolated boundary element 
$g$ corresponding to $\Gamma$. However, a Riemann surface of finite genus has only boundary elements of the first kind, see, e.g., IV.II.6 in [35]. Consequently, $\Gamma$ has a neighborhood $U^{*}$ from the side of $D$ of genus zero with a closed Jordan curve $\gamma=\partial U^{*} \cap D$. Set $U=U^{*} \cup(V \backslash D)$. Correspondingly to the Kerekjarto-Stoilow representation, the latter means that $U^{*}$ is homeomorphic to a plane domain and, consequently, by the general principle of Koebe, see e.g. Section II.3 in [17], $U^{*}$ is conformally equivalent to a plane domain $D^{*}$. Note that by the construction $U^{*}$ had two boundary components. Hence there is a conformal mapping $h$ of $U^{*}$ onto a ring $D^{*}=R=\{z \in \mathbb{C}: 0<r<|z|<1\}$ with $C(\gamma, h)=\{z \in \mathbb{C}:|z|=1\}$ and $C(\Gamma, h)=\{z \in \mathbb{C}:|z|=r\}$, see e.g. Proposition 2.5 in [25] or Proposition 13.5 in [20].

Now, $U^{*}$ and $R$ are Riemann surfaces of hyperbolic type and the modulus $M$ of curve families are invariant under the conformal mapping $h$, see a discussion in [29]- [31]. By condition (i) we have, for a chain $\left\{\sigma_{m}\right\}$ in a prime end $P$ associated with the component $\Gamma$ and localized in $U^{*}$, that

$$
M\left(\Delta\left(\sigma_{m}, \sigma_{m+1}, U^{*}\right)\right)<\infty \quad \forall m=1,2, \ldots
$$

where $\Delta(E, F, G)$ denotes a family of all curves joining the sets $E$ and $F$ through the set $G$. Moreover, by Remark 2.1 the prime end $P$ contains a convergent chain $\left\{\sigma_{m}\right\}$ for which and any continuum $C$ in $U^{*}$

$$
\lim _{m \rightarrow \infty} M\left(\Delta\left(\sigma_{m}, C, U^{*}\right)\right)=0 .
$$

Similarly, prime ends associated with $\gamma$ satisfy conditions (3.1) and (3.2). Thus, the prime ends of $U^{*}$ in the sense (i)-(iii) and their images in $R$ are the prime ends in the sense of Section 4 in [21]. The Näkki prime ends in $R$ has a natural one-to-one correspondence with the points of $\partial R$ whose extension to the correspondence between $\bar{R}$ and $\bar{R}_{P}$ by the identity in $R$ is a homeomorphism with respect to the topologies of $\bar{R}$ and $\bar{R}_{P}$ or with respect to convergence of points and prime ends, correspondingly, see Theorems 4.1 and 4.2 in [21].

Remark 3.1. So, the space of $\bar{U}^{*} P$ with the topology of prime ends is metrizable by $\rho\left(p_{1}, p_{2}\right):=\left|\tilde{h}\left(p_{1}\right)-\tilde{h}\left(p_{2}\right)\right|$, where $\tilde{h}$ is the extension of $h: U^{*} \rightarrow R$ to the homeomorphism $\tilde{h}: \bar{U}^{*} P \rightarrow \bar{R}$ from Lemma 3.1, and the space $\left(\overline{U^{*}} P, \rho\right)$ is compact.

Furthermore, if $D$ be a domain in the Kerekjarto-Stoilow compactification $\overline{\mathbb{S}}$ of a Riemann surface $\mathbb{S}$ and $\partial D$ is a set in $\mathbb{S}$ with a finite collection of components, then the whole space $\bar{D}_{P}$ can be metrized through the theory of pseudometric spaces, see e.g. Section 2.21.XV in [18], and it 
is compact. Namely, let $\rho_{0}$ be one of the metrics on $\overline{\mathbb{S}}$ and let $\rho_{1}, \ldots, \rho_{n}$ be the above metrics on $\overline{U_{1}^{*}}, \ldots, \overline{U_{n}^{*}}$ for the corresponding components $\Gamma_{1}, \ldots, \Gamma_{n}$ of $\partial D$. Then $\rho_{j}^{*}:=\rho_{j} /\left(1+\rho_{j}\right) \leq 1, j=0,1, \ldots, n$, be also metrics generated the same topologies on $\overline{\mathbb{S}}, \overline{U_{1 P}^{*}}, \ldots, \overline{U_{n P}^{*}}$, correspondingly, see e.g. Section 2.21.V in [18]. Then the topology of prime ends on $\bar{D}_{P}$ is generated by the metric $\rho=\sum_{j=0}^{n} 2^{-(j+1)} \tilde{\rho}_{j}<1$ where the pseudometrics $\tilde{\rho}_{j}$ are extensions of $\rho_{j}^{*}$ onto $\bar{D}_{P}$ by 1 , see e.g. Remark 2 in point 2.21.XV of [18].

\section{Some general topological lemmas}

Let us give definitions of topological notions and facts of a general character that will be useful in what follows. Let $T$ be an arbitrary topological space. Then a path in $T$ is a continuous map $\gamma:[a, b] \rightarrow T$. Given $A, B, C \subseteq T, \Delta(A, B, C)$ denotes a collection of all paths $\gamma$ joining $A$ and $B$ in $C$, i.e., $\gamma(a) \in A, \gamma(b) \in B$ and $\gamma(t) \in C$ for all $t \in(a, b)$. In what follows, $|\gamma|$ denotes the locus of $\gamma$, i.e. the image $\gamma([a, b])$.

Proposition 4.1. Let $T$ be a topological space. Suppose that $E_{1}$ and $E_{2}$ are sets in $T$ with $\overline{E_{1}} \cap \overline{E_{2}}=\varnothing$. Then

$$
\Delta\left(E_{1}, E_{2}, T\right)>\Delta\left(\partial E_{1}, \partial E_{2}, T \backslash\left(\bar{E}_{1} \cup \bar{E}_{2}\right)\right) .
$$

Proof. Indeed, let $\gamma \in \Delta\left(E_{1}, E_{2}, T\right)$, i.e. the path $\gamma:[a, b] \rightarrow T$ is such that $\gamma(a) \in E_{1}$ and $\gamma(b) \in E_{2}$. Note that the set $\alpha:=\gamma^{-1}\left(\overline{E_{1}}\right)$ is a closed subset of the segment $[a, b]$ because $\gamma$ is continuous, see e.g. Theorem 1 in Section I.2.1 of [4]. Consequently, $\alpha$ is compact because $[a, b]$ is a compact space, see e.g. I.9.3 in [4]. Then there is $a_{*}:=\max _{t \in \alpha} t<b$ because $\gamma(b) \in E_{2}$ and by the hypothesis of the proposition $\overline{E_{1}} \cap \overline{E_{2}}=\varnothing$. Thus, $\gamma^{\prime}:=\left.\gamma\right|_{\left[a_{*}, b\right]}$ belongs to $\Delta\left(\partial E_{1}, E_{2}, T \backslash \bar{E}_{1}\right)$ because $\gamma$ is continuous and hence $\gamma^{\prime}\left(a_{*}\right)$ cannot be an inner point of $E_{1}$.

Arguing similarly in the space $T^{\prime}=T \backslash E_{1}$ with $E_{1}^{\prime}:=E_{2}$ and $E_{2}^{\prime}:=$ $\partial E_{1}$, we obtain that there is $b_{*}:=\min _{\gamma^{\prime}(t) \in \overline{E_{2}}} t>a_{*}$. Thus, by the given construction $\gamma_{*}:=\left.\gamma\right|_{\left[a_{*}, b_{*}\right]}$ just belongs to $\Delta\left(\partial E_{1}, \partial E_{2}, T \backslash\left(\bar{E}_{1} \cup \bar{E}_{2}\right)\right)$.

Lemma 4.1. In addition to the hypothesis of Proposition 4.1, let $T$ be a subspace of a metric space $(M, \rho)$. Suppose that

$$
\begin{gathered}
\partial E_{1} \subseteq C_{1}:=\left\{p \in M: \rho\left(p, p_{0}\right)=R_{1}\right\}, \\
\partial E_{2} \subseteq C_{2}:=\left\{p \in M: \rho\left(p, p_{0}\right)=R_{2}\right\}
\end{gathered}
$$


with $p_{0} \in M \backslash T$ and $R_{1}<R_{2}$. Then

$$
\Delta\left(E_{1}, E_{2}, T\right)>\Delta\left(C_{1}, C_{2}, A\right)
$$

where

$$
A=A\left(p_{0}, R_{1}, R_{2}\right):=\left\{p \in M: R_{1}<\rho\left(p, p_{0}\right)<R_{2}\right\} .
$$

Note that here, generally speaking, $C_{1} \cap T \neq E_{1}$ and $C_{2} \cap T \neq E_{2}$ as well as $\gamma_{*}$ in the proof of Proposition 4.1 is not in $R$.

Proof. First of all, note that by the continuity of $\gamma_{*}$ the set $\omega:=\gamma_{*}^{-1}(R)$ is open in $\left[a_{*}, b_{*}\right]$ and $\omega$ is the union of a countable collection of disjoint intervals $\left(a_{1}, b_{1}\right),\left(a_{2}, b_{2}\right), \ldots$ with ends in $\Gamma:=\gamma_{*}^{-1}(\partial R)$. If there is a pair $a_{k}$ and $b_{k}$ in the different sets $\Gamma_{i}:=\gamma_{*}^{-1}\left(C_{i}\right), i=1,2, \Gamma=\Gamma_{1} \cup \Gamma_{2}$, $\Gamma_{1} \cap \Gamma_{2}=\varnothing$, then the proof is complete.

Let us assume that such a pair is absent. Then the given collection is split into 2 collections of disjoint intervals $\left(a_{l}^{\prime}, b_{l}^{\prime}\right)$ and $\left(a_{l}^{\prime \prime}, b_{l}^{\prime \prime}\right)$ with ends $a_{l}^{\prime}, b_{l}^{\prime} \in \Gamma_{1}$ and $a_{l}^{\prime \prime}, b_{l}^{\prime \prime} \in \Gamma_{2}, l=1,2, \ldots$. Set $\alpha_{1}=\bigcup_{l}\left(a_{l}^{\prime}, b_{l}^{\prime}\right)$ and $\alpha_{2}=\bigcup_{l}\left(a_{l}^{\prime \prime}, b_{l}^{\prime \prime}\right)$.

Arguing by contradiction, it is easy to show that $\gamma_{*}:\left[a_{*}, b_{*}\right] \rightarrow(M, \rho)$ is uniformly continuous because $\left[a_{*}, b_{*}\right]$ is a compact space. Indeed, let us assume that there is $\varepsilon>0$ and a sequence of pairs $a_{n}^{*}$ and $b_{n}^{*} \in\left[a_{*}, b_{*}\right]$, $n=1,2, \ldots$, such that $\left|b_{n}^{*}-a_{n}^{*}\right| \rightarrow 0$ as $n \rightarrow \infty$ and simultaneously $\rho\left(\gamma_{*}\left(a_{n}^{*}\right), \gamma_{*}\left(b_{n}^{*}\right)\right) \geq \varepsilon$. However, by compactness of $\left[a_{*}, b_{*}\right]$ there is a subsequence $a_{n_{k}}^{*} \rightarrow a_{0} \in\left[a_{*}, b_{*}\right]$ and then also $b_{n_{k}}^{*} \rightarrow a_{0}$ as $k \rightarrow \infty$. Hence by the continuity of $\gamma_{*}$ it should be $\rho\left(\gamma_{*}\left(a_{n_{k}}^{*}\right), \gamma_{*}\left(a_{0}\right)\right) \rightarrow 0$ as well as $\rho\left(\gamma_{*}\left(b_{n_{k}}^{*}\right), \gamma_{*}\left(a_{0}\right)\right) \rightarrow 0$ and then by the triangle inequality also $\rho\left(\gamma_{*}\left(a_{n_{k}}^{*}\right), \gamma_{*}\left(b_{n_{k}}^{*}\right)\right) \rightarrow 0$ as $k \rightarrow \infty$. The contradiction disproves the assumption.

Note that $b_{l}^{\prime}-a_{l}^{\prime} \rightarrow 0$ as $l \rightarrow \infty$ and by the uniform continuity of $\gamma_{*}$ on $\left[a_{*}, b_{*}\right]$ we have that $\left|\gamma_{l}^{\prime}\right| \rightarrow C_{1}$ in the sense that

$$
\sup _{p \in\left|\gamma_{l}^{\prime}\right|} \inf _{q \in C_{1}} \rho(p, q) \rightarrow 0 \quad \text { as } l \rightarrow \infty
$$

where $\gamma_{l}^{\prime}:=\left.\gamma_{*}\right|_{\left[a_{l}^{\prime}, b_{l}^{\prime}\right]}, l=1,2, \ldots$ Thus, there is $R_{2}^{\prime} \in\left(R_{1}, R_{2}\right)$ such that the set $L_{1}:=\bigcup_{l}\left|\gamma_{l}^{\prime}\right|$ lies outside of $B_{2}:=\left\{p \in M: \rho\left(p, p_{0}\right)>R_{2}^{\prime}\right\}$.

Arguing similarly, we obtain that there is $R_{1}^{\prime} \in\left(R_{1}, R_{2}^{\prime}\right)$ such that the set $L_{2}:=\bigcup_{l}\left|\gamma_{l}^{\prime \prime}\right|$ lies outside of $B_{1}:=\left\{p \in M: \rho\left(p, p_{0}\right)<R_{1}^{\prime}\right\}$. Remark that the sets $\beta_{1}:=\gamma_{*}^{-1}\left(B_{1}\right)$ and $\beta_{2}:=\gamma_{*}^{-1}\left(B_{2}\right)$ are open in $\left[a_{*}, b_{*}\right]$ because $\gamma_{*}$ is continuous and by the construction $\delta_{1}:=\alpha_{1} \cup \beta_{1}$ 
and $\delta_{2}:=\alpha_{2} \cup \beta_{2}$ are open, mutually disjoint and together cover the segment $\left[a_{*}, b_{*}\right]$. The latter contradicts to connectedness of the segment and, thus, disproves the above assumption.

\section{On boundary behavior in prime ends of inverse maps}

The main base for extending inverse mappings is the following fact.

Lemma 5.1. Let $\mathbb{S}$ and $\mathbb{S}^{\prime}$ be Riemann surfaces, $D$ and $D^{\prime}$ be domains in $\overline{\mathbb{S}}$ and $\overline{\mathbb{S}^{\prime}}, \partial D \subset \mathbb{S}$ and $\partial D^{\prime} \subset \mathbb{S}^{\prime}$ have finite collections of components, and let $f: D \rightarrow D^{*}$ be a homeomorphism of finite distortion with $K_{f} \in L_{\mathrm{loc}}^{1}$. Then

$$
C\left(P_{1}, f\right) \cap C\left(P_{2}, f\right)=\varnothing
$$

for all prime ends $P_{1} \neq P_{2}$ in the domain $D$.

Here we use the notation of the cluster set of the mapping $f$ at $P \in E_{D}$,

$$
C(P, f):=\left\{P^{\prime} \in E_{D^{\prime}}: P^{\prime}=\lim _{k \rightarrow \infty} f\left(p_{k}\right), p_{k} \rightarrow P, p_{k} \in D\right\} .
$$

As usual, we also assume here that the dilatation $K_{f}$ of the mapping $f$ is extended by zero outside of the domain $D$.

Proof. First of all note that $\overline{\mathbb{S}}$ and $\overline{\mathbb{S}^{\prime}}$ are metrizable spaces. Hence their compactness is equivalent to their sequential compactness, see e.g. Remark 41.I.3 in [19], and, consequently, $\partial D$ and $\partial D^{\prime}$ are compact subsets of $\mathbb{S}$ and $\mathbb{S}^{\prime}$, correspondingly, see e.g. Proposition I.9.3 in [4]. Thus, in view of Remarks 2.1 and 3.1 and Lemma 3.1, we may assume that $\mathbb{S}$ is hyperbolic, $\bar{D}$ is a compact set in $\mathbb{S}, K_{f} \in L^{1}(D), P_{1}$ and $P_{2}$ are associated with the same component $\Gamma$ of $\partial D$ and $D^{\prime}$ is a ring $R=\{z \in \mathbb{C}: 0<r<|z|<1\}$ and

$$
A_{k}:=C\left(P_{k}, f\right), \quad k=1,2
$$

are sets of points in the circle $C_{r}:=\{z \in \mathbb{C}:|z|=r\}, \partial D$ consists of 2 components: $\Gamma$ and a closed Jordan curve $\gamma, C(\gamma, f)=C_{*}:=\{z \in$ $\mathbb{C}:|z|=1\}, C\left(C_{*}, f^{-1}\right)=\gamma, C\left(C_{r}, f^{-1}\right)=\Gamma$, see also Proposition 2.5 in [25] or Proposition 13.5 in [20]. Furthermore, then the sets $A_{k}$ are continua, i.e. closed arcs of the circle $C_{r}$, because

$$
A_{k}=\bigcap_{m=1}^{\infty} \overline{f\left(d_{m}^{(k)}\right)}, \quad k=1,2,
$$


where $d_{m}^{(k)}$ are domains corresponding to chains of cross-cuts $\left\{\sigma_{m}^{(k)}\right\}$ in the prime ends $P_{k}, k=1,2$, see e.g. I $(9.12)$ in [37] and also I.9.3 in [4]. In addition, by Remark 2.1 we may assume also that $\sigma_{m}^{(k)}$ are open arcs of the hyperbolic circles $C_{m}^{(k)}:=\left\{p \in \mathbb{S}: h\left(p, p_{k}\right)=r_{m}^{(k)}\right\}$ on $\mathbb{S}$ with $p_{k} \in \partial D$ and $r_{m}^{(k)} \rightarrow 0$ as $m \rightarrow \infty, k=1,2$.

Set $p_{0}=p_{1}$. By the definition of the topology of the prime ends in the space $\bar{D}_{P}$, we have that $d_{m}^{(1)} \cap d_{m}^{(2)}=\varnothing$ for all large enough $m$ because $P_{1} \neq P_{2}$. For a such $m$, set $R_{1}=r_{m+1}^{(1)}<R_{2}=r_{m}^{(1)}$ and

$$
U_{k}=d_{m}^{(k)}, \quad \Sigma_{k}=\sigma_{m}^{(k)}, \quad C_{k}=\left\{p \in \mathbb{S}: h\left(p, p_{0}\right)=R_{k}\right\}, \quad k=1,2 .
$$

Let $K_{1}$ and $K_{2}$ be arbitrary continua in $U_{1}$ and $U_{2}$, correspondingly. Applying Proposition 4.1 and Lemma 4.1 with $T=D, E_{1}=d_{m+1}^{(1)}$ and $E_{2}=D \backslash d_{m}^{(1)}$, and taking into account the inclusion $\Delta\left(K_{1}, K_{2}, D\right) \subset$ $\Delta\left(E_{1}, E_{2}, D\right)$, we obtain that

$$
\Delta\left(K_{1}, K_{2}, D\right)>\Delta\left(C_{1}, C_{2}, A\right), \quad A:=\left\{p \in \mathbb{S}: R_{1}<h\left(p, p_{0}\right)<R_{2}\right\},
$$

which means that any path $\alpha:[a, b] \rightarrow \mathbb{S}$ joining $K_{1}$ and $K_{2}$ in $D$, $\alpha(a) \in K_{1}, \alpha(b) \in K_{2}$ and $\alpha(t) \in D, t \in(a, b)$, has a subpath joining $C_{1}$ and $C_{2}$ in $A$. Thus, since $f$ is a homeomorphism, we have also that

$$
\Delta\left(f K_{1}, f K_{2}, f D\right)>\Delta\left(f C_{1}, f C_{2}, f A\right)
$$

and by the minorization principle, see e.g. [7, p. 178], we obtain that

$$
M\left(\Delta\left(f K_{1}, f K_{2}, f D\right)\right) \leq M\left(\Delta\left(f C_{1}, f C_{2}, f A\right)\right) .
$$

So, by Lemma 3.1 in [30] and [31] we conclude that

$$
M\left(\Delta\left(f K_{1}, f K_{2}, f D\right)\right) \leqslant \int_{A} K_{f}(p) \cdot \xi^{2}\left(h\left(p, p_{0}\right)\right) d h(p)
$$

for all measurable functions $\xi:\left(R_{1}, R_{2}\right) \rightarrow[0, \infty]$ such that

$$
\int_{R_{1}}^{R_{2}} \xi(R) d R \geqslant 1 .
$$

In particular, for $\xi(R) \equiv 1 / \delta, \delta=R_{2}-R_{1}>0$, we get from here that

$$
M\left(\Delta\left(f K_{1}, f K_{2}, f D\right)\right) \leqslant M_{0}:=\frac{1}{\delta} \int_{D} K_{f}(p) d h(p)<\infty .
$$


Since $f$ is a homeomorphism, (5.7) means that

$$
M\left(\Delta\left(\mathcal{K}_{1}, \mathcal{K}_{2}, D^{\prime}\right)\right) \leqslant M_{0}<\infty
$$

for all continua $\mathcal{K}_{1}$ and $\mathcal{K}_{2}$ in the domains $V_{1}=f U_{1}$ and $V_{2}=f U_{2}$, correspondingly.

Let us assume that $A_{1} \cap A_{2} \neq \varnothing$. Then by the construction there is $p_{0} \in \partial R \cap \partial V_{1} \cap \partial V_{2}$. However, the latter contradicts (5.8) because the ring $R$ is a $\mathrm{QED}$ (quasiextremal distance) domains, see e.g. Theorem 3.2 in [20], see also Theorem 10.12 in [36].

Theorem 5.1. Let $\mathbb{S}$ and $\mathbb{S}^{\prime}$ be Riemann surfaces, $D$ and $D^{\prime}$ be domains in $\overline{\mathbb{S}}$ and $\overline{\mathbb{S}^{\prime}}$, correspondingly, $\partial D \subset \mathbb{S}$ and $\partial D^{\prime} \subset \mathbb{S}^{\prime}$ have finite collections of nondegenerate components, and let $f: D \rightarrow D^{\prime}$ be a homeomorphism of finite distortion with $K_{f} \in L_{\mathrm{loc}}^{1}$. Then the inverse mapping $g=f^{-1}: D^{\prime} \rightarrow D$ can be extended to a continuous mapping $\tilde{g}$ of ${\overline{D^{\prime}}}_{P}$ onto $\bar{D}_{P}$.

Proof. Recall that by Remark 3.1 the spaces $\bar{D}_{P}$ and ${\overline{D^{\prime}}}_{P}$ are compact and metrizable with metrics $\rho$ and $\rho^{\prime}$. Let a sequence $p_{n} \in D^{\prime}$ converges as $n \rightarrow \infty$ to a prime end $P^{\prime} \in E_{D^{\prime}}$. Then any subsequence of $p_{n}^{*}:=g\left(p_{n}\right)$ has a convergent subsequence by compactness of $\bar{D}_{P}$. By Lemma 5.1 any such convergent subsequence should have the same limit. Thus, the sequence $p_{n}^{*}$ is convergent, see e.g. Theorem 2 of Section 2.20.II in [18]. Note that $p_{n}^{*}$ cannot converge to an inner point of $D$ because $I(P) \subseteq \partial D$ by Proposition 2.1 and, consequently, $p_{n}$ is convergent to $\partial D^{\prime}$, see e.g. Proposition 2.5 in [25] or Proposition 13.5 in [20]. Thus, $E_{D^{\prime}}$ is mapped into $E_{D}$ under this extension $\tilde{g}$ of $g$. In fact, $\tilde{g}$ maps $E_{D^{\prime}}$ onto $E_{D}$ because $p_{n}=f\left(p_{n}^{*}\right)$ has a convergent subsequence for every sequence $p_{n}^{*} \in D$ that is convergent to a prime end $P$ of the domain $D$ because $\overline{D^{\prime}} P$ is compact. The map $\tilde{g}$ is continuous. Indeed, let a sequence $P_{n}^{\prime} \in \overline{D^{\prime}}{ }_{P}$ be convergent to $P^{\prime} \in{\overline{D^{\prime}}}_{P}$. Then there is a sequence $p_{n} \in D^{\prime}$ such that $\rho^{\prime}\left(P_{n}^{\prime}, p_{n}\right)<2^{-n}$ and $\rho\left(p_{n}^{*}, P_{n}^{*}\right)<2^{-n}$ where $p_{n}^{*}:=g\left(p_{n}\right), P_{n}^{*}:=\tilde{g}\left(P_{n}\right)$ and $P^{*}=\tilde{g}\left(P^{\prime}\right)$. Then $p_{n} \rightarrow P^{\prime}$ and by the above $p_{n}^{*} \rightarrow P^{*}$ as well as $P_{n}^{*} \rightarrow P^{*}$ as $n \rightarrow \infty$.

\section{Lemma on extension to boundary of direct mappings}

In contrast with the case of the inverse mappings, as it was already established in the plane, no degree of integrability of the dilatation leads to the extension to the boundary of direct mappings with finite distortion, see the example in the proof of Proposition 6.3 in [20]. The nature of the corresponding conditions has a much more refined character as the following lemma demonstrates. 
Lemma 6.1. Under the hypothesis of Theorem 5.1, let in addition

$$
\int_{R\left(p_{0}, \varepsilon, \varepsilon_{0}\right)} K_{f}(p) \cdot \psi_{p_{0}, \varepsilon, \varepsilon_{0}}^{2}\left(h\left(p, p_{0}\right)\right) d h(p)=o\left(I_{p_{0}, \varepsilon_{0}}^{2}(\varepsilon)\right) \quad \forall p_{0} \in \partial D
$$

as $\varepsilon \rightarrow 0$ for all $\varepsilon_{0}<\delta\left(p_{0}\right)$ where $R\left(p_{0}, \varepsilon, \varepsilon_{0}\right)=\left\{p \in \mathbb{S}: \varepsilon<h\left(p, p_{0}\right)<\right.$ $\left.\varepsilon_{0}\right\}$ and $\psi_{p_{0}, \varepsilon, \varepsilon_{0}}(t):(0, \infty) \rightarrow[0, \infty], \varepsilon \in\left(0, \varepsilon_{0}\right)$, is a family of measurable functions such that

$$
0<I_{p_{0}, \varepsilon_{0}}(\varepsilon):=\int_{\varepsilon}^{\varepsilon_{0}} \psi_{p_{0}, \varepsilon, \varepsilon_{0}}(t) d t<\infty \quad \forall \varepsilon \in\left(0, \varepsilon_{0}\right) .
$$

Then $f$ can be extended to a continuous mapping $\tilde{f}$ of $\bar{D}_{P}$ onto $\overline{D^{\prime}}$.

We assume here that the function $K_{f}$ is extended by zero outside of $D$.

Proof. By Remarks 2.1 and 3.1 and Lemma 3.1, arguing as in the beginning of the proof of Lemma 5.1, we may assume that $\bar{D}$ is a compact set in $\mathbb{S}, \partial D$ consists of 2 components: a closed Jordan curve $\gamma$ and one more nondegenerate component $\Gamma, D^{\prime}$ is a ring $R=\{z \in \mathbb{C}: 0<r<|z|<1\}$, ${\overline{D^{\prime}}}_{P}=\bar{R}$,

$$
C(\Gamma, f)=C_{r}:=\{z \in \mathbb{C}:|z|=r\}, \quad C(\gamma, f)=C_{*}:=\{z \in \mathbb{C}:|z|=1\}
$$

and that $f$ is extended to a homeomorphism of $D \cup \gamma$ onto $D^{\prime} \cup C_{*}$.

Let us first prove that the set $L:=C(P, f)$ consists of a single point of $C_{r}$ for a prime end $P$ of the domain $D$ associated with $\Gamma$. Note that $L \neq \varnothing$ by compactness of the set $\bar{R}$ and, moreover, $L \subseteq C_{r}$ by Proposition 2.1 .

Let us assume that there is at least two points $\zeta_{0}$ and $\zeta_{*} \in L$. Set $U=\left\{\zeta \in \mathbb{C}:\left|\zeta-\zeta_{0}\right|<\rho_{0}\right\}$ where $0<\rho_{0}<\left|\zeta_{*}-\zeta_{0}\right|$.

Let $\sigma_{k}, k=1,2, \ldots$, be a chain in the prime end $P$ from Remark 2.1 lying on the circles $S_{k}:=\left\{p \in \mathbb{S}: h\left(p, p_{0}\right)=r_{k}\right\}$ where $p_{0} \in \Gamma$ and $r_{k} \rightarrow 0$ as $k \rightarrow \infty$. Let $d_{k}$ be the domains associated with $\sigma_{k}$. Then there exist points $\zeta_{k}$ and $\zeta_{k}^{*}$ in the domains $d_{k}^{\prime}=f\left(d_{k}\right) \subset R$ such that $\left|\zeta_{0}-\zeta_{k}\right|<\rho_{0}$ and $\left|\zeta_{0}-\zeta_{k}^{*}\right|>\rho_{0}$ and, moreover, $\zeta_{k} \rightarrow \zeta_{0}$ and $\zeta_{k}^{*} \rightarrow \zeta_{*}$ as $k \rightarrow \infty$. Let $\gamma_{k}$ be paths joining $\zeta_{k}$ and $\zeta_{k}^{*}$ in $d_{k}^{\prime}$. Note that by the construction $\partial U \cap \gamma_{k} \neq \varnothing, k=1,2, \ldots$

By the condition of strong accessibility of the point $\zeta_{0}$ in the ring $R$, there is a continuum $E \subset R$ and a number $\delta>0$ such that

$$
M\left(\Delta\left(E, \gamma_{k} ; R\right)\right) \geqslant \delta
$$


for all large enough $k$. Note that $C=f^{-1}(E)$ is a compact subset of $D$ and hence $\left.h\left(p_{0}, C\right)\right)>0$. Let $\varepsilon_{0} \in\left(0, \delta_{0}\right)$ where $\delta_{0}:=\min \left(\delta\left(p_{0}\right), h\left(p_{0}, C\right)\right)$. Without loss of generality, we may assume that $r_{k}<\varepsilon_{0}$ and that (6.2) holds for all $k=1,2, \ldots$.

Let $\Gamma_{m}$ be the family of paths joining the circle $S_{0}:=\{p \in \mathbb{S}$ : $\left.h\left(p, p_{0}\right)=\varepsilon_{0}\right\}$ and $\sigma_{m}, m=1,2, \ldots$, in the intersection of $D \backslash d_{m}$ and the ring $R_{m}:=\left\{p \in \mathbb{S}: r_{m}<h\left(p, p_{0}\right)<\varepsilon_{0}\right\}$. Applying Proposition 4.1 and Lemma 4.1 with $T=D, E_{1}=d_{m}$ and $E_{2}=B_{0}:=\left\{p \in \mathbb{S}: h\left(p, p_{0}\right)>\right.$ $\left.\varepsilon_{0}\right\}$, and taking into account the inclusion $\Delta\left(C, C_{k}, D\right) \subset \Delta\left(E_{1}, E_{2}, D\right)=$ $\Delta\left(B_{0}, d_{m}, D\right)$ where $C_{k}=f^{-1}\left(\gamma_{k}\right)$, we have that $\Delta\left(C, C_{k}, D\right)>\Gamma_{m}$ for all $k \geqslant m$ because by the construction $C_{k} \subset d_{k} \subset d_{m}$. Thus, since $f$ is a homeomorphism, we have also that $\Delta\left(E, \gamma_{k}, D\right)>f \Gamma_{m}$ for all $k \geqslant m$, and by the principle of minorization, see e.g. [7], p. 178, we obtain that $M\left(f\left(\Gamma_{m}\right)\right) \geqslant \delta$ for all $m=1,2, \ldots$.

On the other hand, every function

$$
\xi(t)=\xi_{m}(t):=\psi_{p_{0}, r_{m}, \varepsilon_{0}}(t) / I_{p_{0}, \varepsilon_{0}}\left(r_{m}\right), \quad m=1,2, \ldots,
$$

satisfies the condition (5.6) and by Lemma 3.1 in [30] and [31]

$$
M\left(f \Gamma_{m}\right) \leqslant \int_{R_{m}} K_{f}(p) \cdot \xi_{m}^{2}\left(h\left(p, p_{0}\right)\right) d h(p),
$$

i.e., $M\left(f \Gamma_{m}\right) \rightarrow 0$ as $m \rightarrow \infty$ in view of (6.1).

The obtained contradiction disproves the assumption that the cluster set $C(P, f)$ consists of more than one point.

Thus, we have the extension $\tilde{f}$ of $f$ to $\bar{D}_{P}$ such that $\tilde{f}\left(E_{D}\right) \subseteq E_{D^{\prime}}$. In fact, $\tilde{f}\left(E_{D}\right)=E_{D^{\prime}}$. Indeed, if $\zeta_{0} \in D^{\prime}$, then there is a sequence $\zeta_{n}$ in $D^{\prime}$ that is convergent to $\zeta_{0}$. We may assume with no loss of generality that $f^{-1}\left(\zeta_{n}\right) \rightarrow P_{0} \in \bar{D}_{P}$ because $\bar{D}_{P}$ is compact, see Remark 3.1. Hence $\zeta_{0} \in E_{D}$ because $\zeta_{0} \notin D$, see e.g. Proposition 2.5 in [25] or Proposition 13.5 in [20].

Finally, let us show that the extended mapping $\tilde{f}: \bar{D}_{P} \rightarrow{\overline{D^{\prime}}}_{P}$ is continuous. Indeed, let $P_{n} \rightarrow P_{0}$ in $\bar{D}_{P}$. The statement is obvious for $P_{0} \in D$. If $P_{0} \in E_{D}$, then by the last item we are able to choose $P_{n}^{*} \in D$ such that $\rho\left(P_{n}, P_{n}^{*}\right)<2^{-n}$ and $\rho^{\prime}\left(\tilde{f}\left(P_{n}\right), \tilde{f}\left(P_{n}^{*}\right)\right)<2^{-n}$ where $\rho$ and $\rho^{\prime}$ are some metrics on $\bar{D}_{P}$ and ${\overline{D^{\prime}}}_{P}$, correspondingly, see Remark 3.1. Note that by the first part of the proof $f\left(P_{n}^{*}\right) \rightarrow f\left(P_{0}\right)$ because $P_{n}^{*} \rightarrow P_{0}$. Consequently, $\tilde{f}\left(P_{n}\right) \rightarrow \tilde{f}\left(P_{0}\right)$, too.

Remark 6.1. Note that condition (6.1) holds, in particular, if

$$
\int_{D\left(p_{0}, \varepsilon_{0}\right)} K_{f}(p) \cdot \psi^{2}\left(h\left(p, p_{0}\right)\right) d h(p)<\infty \quad \forall p_{0} \in \partial D
$$


where $D\left(p_{0}, \varepsilon_{0}\right)=\left\{p \in \mathbb{S}: h\left(p, p_{0}\right)<\varepsilon_{0}\right\}$ and where $\psi(t):(0, \infty) \rightarrow$ $[0, \infty]$ is a locally integrable function such that $I_{p_{0}, \varepsilon_{0}}(\varepsilon) \rightarrow \infty$ as $\varepsilon \rightarrow 0$. In other words, for the extendability of $f$ to a continuous mapping of $\bar{D}_{P}$ onto $\overline{D^{\prime}}{ }_{P}$, it suffices for the integrals in (6.3) to be convergent for some nonnegative function $\psi(t)$ that is locally integrable on $(0, \infty)$ but that has a non-integrable singularity at zero.

\section{On the homeomorphic extension to the boundary}

Combining Lemma 6.1 and Theorem 5.1, we obtain the significant conclusion:

Lemma 7.1. Under the hypothesis of Lemma 6.1, the homeomorphism $f: D \rightarrow D^{\prime}$ can be extended to a homeomorphism $\tilde{f}: \bar{D}_{P} \rightarrow{\overline{D^{\prime}}}_{P}$.

Proof. Indeed, by Lemma 5.1 the mapping $\tilde{f}: \bar{D}_{P} \rightarrow{\overline{D^{\prime}}}_{P}$ from Lemma 6.1 is injective and hence it has the well defined inverse mapping $\tilde{f}^{-1}$ : ${\overline{D^{\prime}}}_{P} \rightarrow \bar{D}_{P}$ and the latter coincides with the mapping $\tilde{g}:{\overline{D^{\prime}}}_{P} \rightarrow \bar{D}_{P}$ from Theorem 5.1 because a limit under a metric convergence is unique. The continuity of the mappings $\tilde{g}$ and $\tilde{f}$ follows from Theorem 5.1 and Lemma 6.1, respectively.

We assume everywhere in this section that the function $K_{f}$ is extended by zero outside of $D$.

Theorem 7.1. Under the hypothesis of Theorem 5.1, let in addition

$$
\int_{0}^{\varepsilon_{0}} \frac{d r}{\left\|K_{f}\right\|\left(p_{0}, r\right)}=\infty \quad \forall p_{0} \in \partial D, \quad \varepsilon_{0}<\delta\left(p_{0}\right)
$$

where

$$
\left\|K_{f}\right\|\left(p_{0}, r\right):=\int_{S\left(p_{0}, r\right)} K_{f}(p) d s_{h}(p) .
$$

Then $f$ can be extended to a homeomorphism of $\bar{D}_{P}$ onto ${\overline{D^{\prime}}}_{P}$.

Here $S\left(p_{0}, r\right)$ denotes the circle $\left\{p \in \mathbb{S}: h\left(p, p_{0}\right)=r\right\}$.

Proof. Indeed, for the functions

$$
\psi_{p_{0}, \varepsilon_{0}}(t):=\left\{\begin{array}{lr}
1 /\left\|K_{f}\right\|\left(p_{0}, t\right), & t \in\left(0, \varepsilon_{0}\right), \\
0, & t \in\left[\varepsilon_{0}, \infty\right),
\end{array}\right.
$$


we have by the Fubini theorem that

$$
\int_{R\left(p_{0}, \varepsilon, \varepsilon_{0}\right)} K_{f}(p) \cdot \psi_{p_{0}, \varepsilon_{0}}^{2}\left(h\left(p, p_{0}\right) d h(p)=\int_{\varepsilon}^{\varepsilon_{0}} \frac{d r}{\left\|K_{f}\right\|\left(p_{0}, r\right)}\right.
$$

where $R\left(p_{0}, \varepsilon, \varepsilon_{0}\right)$ denotes the $\operatorname{ring}\left\{p \in \mathbb{S}: \varepsilon<h\left(p, p_{0}\right)<\varepsilon_{0}\right\}$ and, consequently, condition (6.1) holds by (7.1) for all $p_{0} \in \partial D$ and $\varepsilon_{0} \in$ $\left(0, \varepsilon\left(p_{0}\right)\right)$.

Here we have used the standard conventions in the integral theory that $a / \infty=0$ for $a \neq \infty$ and $0 \cdot \infty=0$, see, e.g., Section I.3 in [33].

Thus, Theorem 7.1 follows immediately from Lemma 7.1.

Corollary 7.1. In particular, the conclusion of Theorem 7.1 holds if

$$
k_{p_{0}}(r)=O\left(\log \frac{1}{r}\right) \quad \forall p_{0} \in \partial D
$$

as $r \rightarrow 0$ where $k_{p_{0}}(r)$ is the average of $K_{f}$ over the infinitesimal circle $S\left(p_{0}, r\right)$.

Choosing in $(6.1) \psi(t):=\frac{1}{t \log 1 / t}$, we obtain by Lemma 7.1 the next result, see also Lemma 4.1 in [25] or Lemma 13.2 in [20].

Theorem 7.2. Under the hypothesis of Theorem 5.1, let $K_{f}$ have a dominant $Q_{p_{0}}$ in a neighborhood of each point $p_{0} \in \partial D$ with finite mean oscillation at $p_{0}$. Then $f$ can be extended to a homeomorphism $\tilde{f}: \bar{D}_{P} \rightarrow$ $\bar{D}_{P}$.

By Corollary 4.1 in [25] or Corollary 13.3 in [20] we obtain the following.

Corollary 7.2. In particular, the conclusion of Theorem 7.2 holds if

$$
\varlimsup_{\varepsilon \rightarrow 0} f_{D\left(p_{0}, \varepsilon\right)} K_{f}(p) d h(p)<\infty \quad \forall p_{0} \in \partial D
$$

where $D\left(p_{0}, \varepsilon\right)$ is the infinitesimal disk $\left\{p \in \mathbb{S}: h\left(p, p_{0}\right)<\varepsilon\right\}$.

Corollary 7.3. The conslusion of Theorem 7.2 holds if every point $p_{0} \in$ $\partial D$ is a Lebesgue point of the function $K_{f}$ or its dominant $Q_{p_{0}}$.

The next statement also follows from Lemma 7.1 under the choice $\psi(t)=1 / t$. 
Theorem 7.3. Under the hypothesis of Theorem 5.1, let, for some $\varepsilon_{0}>$ 0 ,

$$
\int_{\varepsilon<h\left(p, p_{0}\right)<\varepsilon_{0}} K_{f}(p) \frac{d h(p)}{h^{2}\left(p, p_{0}\right)}=o\left(\left[\log \frac{1}{\varepsilon}\right]^{2}\right) \quad \text { as } \varepsilon \rightarrow 0 \quad \forall p_{0} \in \partial D
$$

Then $f$ can be extended to a homeomorphism of $\bar{D}_{P}$ onto ${\overline{D^{\prime}}}_{P}$.

Remark 7.1. Choosing in Lemma 7.1 the function $\psi(t)=1 /(t \log 1 / t)$ instead of $\psi(t)=1 / t,(7.7)$ can be replaced by the more weak condition

$$
\int_{\varepsilon<h\left(p, p_{0}\right)<\varepsilon_{0}} \frac{K_{f}(p) d h(p)}{\left(h\left(p, p_{0}\right) \log \frac{1}{h\left(p, p_{0}\right)}\right)^{2}}=o\left(\left[\log \log \frac{1}{\varepsilon}\right]^{2}\right)
$$

and (7.5) by the condition

$$
k_{p_{0}}(r)=o\left(\log \frac{1}{\varepsilon} \log \log \frac{1}{\varepsilon}\right) .
$$

Of course, we could give here the whole scale of the corresponding condition of the logarithmic type using suitable functions $\psi(t)$.

\section{On interconnections between integral conditions}

For every non-decreasing function $\Phi:[0, \infty] \rightarrow[0, \infty]$, the inverse function $\Phi^{-1}$ can be well defined by setting

$$
\Phi^{-1}(\tau)=\inf _{\Phi(t) \geq \tau} t .
$$

As usual, here inf is equal to $\infty$ if the set of $t \in[0, \infty]$ such that $\Phi(t) \geq \tau$ is empty. Note that the function $\Phi^{-1}$ is non-decreasing, too.

Remark 8.1. Immediately by the definition it is evident that

$$
\Phi^{-1}(\Phi(t)) \leq t \quad \forall t \in[0, \infty]
$$

with the equality in (8.2) except intervals of constancy of the function $\Phi(t)$.

Recall that a function $\Phi:[0, \infty] \rightarrow[0, \infty]$ is called convex if

$$
\Phi\left(\lambda t_{1}+(1-\lambda) t_{2}\right) \leq \lambda \Phi\left(t_{1}\right)+(1-\lambda) \Phi\left(t_{2}\right)
$$

for all $t_{1}, t_{2} \in[0, \infty]$ and $\lambda \in[0,1]$. 
In what follows, $\mathbb{H}(R)$ denotes the hyperbolic disk centered at the origin with the hyperbolic radius $R=\log (1+r) /(1-r), r \in(0,1)$ is its Euclidean radius:

$$
\mathbb{H}(R)=\{z \in \mathbb{C}: h(z, 0)<R\}, \quad R \in(0, \infty) .
$$

Further we also use the notation of the hyperbolic $\operatorname{sine}: \sinh t:=$ $\left(e^{t}-e^{-t}\right) / 2$.

The following statement is an analog of Lemma 3.1 in [28] adopted to the hyperbolic geometry in the unit disk $\mathbb{D}:=\{z \in \mathbb{C}:|z|<1\}$.

Lemma 8.1. Let $Q: \mathbb{H}(\varepsilon) \rightarrow[0, \infty], \varepsilon \in(0,1)$, be a measurable function and $\Phi:[0, \infty] \rightarrow(0, \infty]$ be a non-decreasing convex function with a finite mean integral value $M(\varepsilon)$ of the function $\Phi \circ Q$ on $\mathbb{H}(\varepsilon)$. Then

$$
\int_{0}^{\varepsilon} \frac{d \rho}{\rho q(\rho)} \geq \frac{1}{2} \int_{\delta(\varepsilon)}^{\infty} \frac{d \tau}{\tau\left[\Phi^{-1}(\tau)\right]}
$$

where $q(\rho)$ is the average of $Q$ on the circle $\mathbb{S}(\rho)=\{z \in \mathbb{D}: h(z, 0)=\rho\}$ and

$$
\delta(\varepsilon)=\exp \left(4 \sinh ^{2} \frac{\varepsilon}{2}\right) \cdot \frac{M(\varepsilon)}{\varepsilon^{2}}>\tau_{0}:=\Phi(0)>0 .
$$

Proof. Since $M(\varepsilon)<\infty$ we may assume with no loss of generality that $\Phi(t)<\infty$ for all $t \in[0, \infty)$ because in the contrary case $Q \in L^{\infty}$ and then the left-hand side in (8.4) is equal to $\infty$. Moreover, we may assume that $\Phi(t)$ is not constant because in the contrary case $\Phi^{-1}(\tau) \equiv \infty$ for all $\tau>\tau_{0}$ and hence the right-hand side in (8.4) is equal to 0 . Note also that $\Phi(\tau)$ is (strictly) increasing, convex and continuous in the segment $\left[t_{*}, \infty\right]$ and

$$
\Phi(t) \equiv \tau_{0} \quad \forall t \in\left[0, t_{*}\right] \quad \text { where } t_{*}:=\sup _{\Phi(t)=\tau_{0}} t .
$$

Setting $H(t):=\log \Phi(t)$, we see that $H^{-1}(\eta)=\Phi^{-1}\left(e^{\eta}\right), \Phi^{-1}(\tau)=$ $H^{-1}(\log \tau)$. Thus, we obtain that

$$
q(\rho)=H^{-1}\left(\log \frac{h(\rho)}{\rho^{2}}\right)=H^{-1}\left(2 \log \frac{1}{\rho}+\log h(\rho)\right) \quad \forall \rho \in R_{*}
$$

where $h(\rho):=\rho^{2} \Phi(q(\rho))$ and $R_{*}=\left\{\rho \in(0, \varepsilon): q(\rho)>t_{*}\right\}$. Then also

$$
q\left(e^{-s}\right)=H^{-1}\left(2 s+\log h\left(e^{-s}\right)\right) \quad \forall s \in S_{*}
$$


where $S_{*}=\left\{s \in\left(\log \frac{1}{\varepsilon}, \infty\right): q\left(e^{-s}\right)>t_{*}\right\}$.

Now, by the Jensen inequality, see e.g. Theorem 2.6.2 in [24], we have that

$$
\begin{aligned}
& \int_{\log \frac{1}{\varepsilon}}^{\infty} h\left(e^{-s}\right) d s=\int_{0}^{\varepsilon} h(\rho) \frac{d \rho}{\rho}=\int_{0}^{\varepsilon} \Phi(q(\rho)) \rho d \rho \\
\leq & \int_{0}^{\varepsilon}\left(f_{S(\rho)} \Phi(Q(z)) d s_{h}(z)\right) \rho d \rho \leq 2 \sinh ^{2} \frac{\varepsilon}{2} \cdot M(\varepsilon)
\end{aligned}
$$

because $\mathbb{H}(\varepsilon)$ has the hyperbolic area $A(\varepsilon)=4 \pi \sinh ^{2} \frac{\varepsilon}{2}$ and $\mathbb{S}(\rho)$ has the hyperbolic length $L(\rho)=2 \pi \sinh \rho$, see e.g. Theorem 7.2.2 in [2], and, moreover, $\sinh \rho \geq \rho$ by the Taylor expansion. Then arguing by contradiction it is easy to see for the set $T:=\left\{s \in\left(\log \frac{1}{\varepsilon}, \infty\right)\right.$ : $\left.h\left(e^{-s}\right)>M(\varepsilon)\right\}$ that its length

$$
|T|=\int_{T} d s \leq 2 \sinh ^{2} \frac{\varepsilon}{2} .
$$

Next, let us show for $T_{*}:=T \cap S_{*}$ that

$$
q\left(e^{-s}\right) \leq H^{-1}(2 s+\log M(\varepsilon)) \quad \forall s \in\left(\log \frac{1}{\varepsilon}, \infty\right) \backslash T_{*} .
$$

Indeed, note that $\left(\log \frac{1}{\varepsilon}, \infty\right) \backslash T_{*}=\left[\left(\log \frac{1}{\varepsilon}, \infty\right) \backslash S_{*}\right] \cup\left[\left(\log \frac{1}{\varepsilon}, \infty\right) \backslash T\right]=$ $\left[\left(\log \frac{1}{\varepsilon}, \infty\right) \backslash S_{*}\right] \cup\left[S_{*} \backslash T\right]$. The inequality (8.11) holds for $s \in S_{*} \backslash T$ by (8.8) because $H^{-1}$ is a non-decreasing function. Note also that

$$
e^{2 s} M(\varepsilon)>\Phi(0)=\tau_{0} \quad \forall s \in\left(\log \frac{1}{\varepsilon}, \infty\right)
$$

and then

$$
t_{*}<\Phi^{-1}\left(e^{2 s} M(\varepsilon)\right)=H^{-1}(2 s+\log M(\varepsilon)) \quad \forall s \in\left(\log \frac{1}{\varepsilon}, \infty\right)
$$

Consequently, (8.11) holds for all $s \in\left(\log \frac{1}{\varepsilon}, \infty\right) \backslash S_{*}$, too.

Since $H^{-1}$ is non-decreasing, we have by (8.10)-(8.11) that, for $\Delta:=$ $\log M(\varepsilon)$,

$$
\int_{0}^{\varepsilon} \frac{d \rho}{\rho q(\rho)}=\int_{\log \frac{1}{\varepsilon}}^{\infty} \frac{d s}{q\left(e^{-s}\right)} \geq \int_{\left(\log \frac{1}{\varepsilon}, \infty\right) \backslash T_{*}} \frac{d s}{H^{-1}(2 s+\Delta)}
$$




$$
\begin{aligned}
\geq \int_{\left|T_{*}\right|+\log \frac{1}{\varepsilon}}^{\infty} \frac{d s}{H^{-1}(2 s+\Delta)} & \geq \int_{2 \sinh ^{2} \frac{\varepsilon}{2}+\log \frac{1}{\varepsilon}}^{\infty} \frac{d s}{H^{-1}(2 s+\Delta)} \\
& =\frac{1}{2} \int_{4 \sinh ^{2} \frac{\varepsilon}{2}+\log \frac{M(\varepsilon)}{\varepsilon^{2}}}^{\infty} \frac{d \eta}{H^{-1}(\eta)}
\end{aligned}
$$

and after the replacement of variables $\eta=\log \tau, \tau=e^{\eta}$, we come to (8.4).

Theorem 8.1. Let $Q: \mathbb{H}(\varepsilon) \rightarrow[0, \infty], \varepsilon \in(0,1)$, be a measurable function such that

$$
\int_{\mathbb{H}(\varepsilon)} \Phi(Q(z)) d h(z)<\infty
$$

where $\Phi:[0, \infty] \rightarrow[0, \infty]$ is a non-decreasing convex function with

$$
\int_{\delta_{0}}^{\infty} \frac{d \tau}{\tau \Phi^{-1}(\tau)}=\infty
$$

for some $\delta_{0}>\tau_{0}:=\Phi(0)$. Then

$$
\int_{0}^{\varepsilon} \frac{d \rho}{\rho q(\rho)}=\infty
$$

where $q(\rho)$ is the average of $Q$ on the hyperbolic circle $h(z, 0)=\rho$.

Proof. If $\Phi(0) \neq 0$, then Theorem 8.1 directly follows from Lemma 8.1 because $\Phi^{-1}$ is strictly increasing on the interval $\left(\tau_{0}, \infty\right)$ and $\Phi^{-1}\left(\delta_{0}\right)>0$. In the case $\Phi(0)=0$, let us fix a number $\delta \in\left(0, \delta_{0}\right)$ and set $\Phi_{*}(t)=\Phi(t)$, if $\Phi(t)>\delta$, and $\Phi_{*}(t)=\delta$, if $\Phi(t) \leq \delta$. Then by (8.15) we have that $\int_{\mathbb{H}(\varepsilon)} \Phi_{*}(Q(z)) d h(z)<\infty$ because $\left|\Phi_{*}(t)-\Phi(t)\right| \leq \delta$ and the measure of $\mathbb{H}(\varepsilon)$ is finite. Moreover, $\Phi_{*}^{-1}(\tau)=\Phi^{-1}(\tau)$ for $\tau \geq \delta$ and then by (8.16) $\int_{\delta_{0}}^{\infty} \frac{d \tau}{\tau \Phi_{*}^{-1}(\tau)}=\infty$. Thus, (8.17) holds again by Lemma 8.1.

Remark 8.2. Note that condition (8.16) implies that

$$
\int_{\delta}^{\infty} \frac{d \tau}{\tau \Phi^{-1}(\tau)}=\infty \quad \forall \delta \in[0, \infty) .
$$


but relation (8.18) for some $\delta \in[0, \infty)$, generally speaking, does not imply (8.16). Indeed, (8.16) evidently implies (8.18) for $\delta \in\left[0, \delta_{0}\right)$, and, for $\delta \in\left(\delta_{0}, \infty\right)$, we have that

$$
0 \leq \int_{\delta_{0}}^{\delta} \frac{d \tau}{\tau \Phi^{-1}(\tau)} \leq \frac{1}{\Phi^{-1}\left(\delta_{0}\right)} \log \frac{\delta}{\delta_{0}}<\infty
$$

because the function $\Phi^{-1}$ is non-decreasing and $\Phi^{-1}\left(\delta_{0}\right)>0$. Moreover, by the definition of the inverse function $\Phi^{-1}(\tau) \equiv 0$ for all $\tau \in\left[0, \tau_{0}\right]$, $\tau_{0}=\Phi(0)$, and hence $(8.18)$ for $\delta \in\left[0, \tau_{0}\right)$, generally speaking, does not imply (8.16). If $\tau_{0}>0$, then

$$
\int_{\delta}^{\tau_{0}} \frac{d \tau}{\tau \Phi^{-1}(\tau)}=\infty \quad \forall \delta \in\left[0, \tau_{0}\right)
$$

However, relation (8.20) gives no information on the function $Q$ itself and, consequently, (8.18) for $\delta<\Phi(0)$ cannot imply (8.17) at all.

\section{Other criteria for homeomorphic extension in prime ends}

Theorem 7.1 has a magnitude of other consequences thanking to Theorem 8.1.

Theorem 9.1. Under the hypothesis of Theorem 5.1, let

$$
\int_{D\left(p_{0}, \varepsilon_{0}\right)} \Phi_{p_{0}}\left(K_{f}(p)\right) d h(p)<\infty \quad \forall p_{0} \in \partial D
$$

for $\varepsilon_{0}=\varepsilon\left(p_{0}\right)$ and a nondecreasing convex function $\Phi_{p_{0}}:[0, \infty) \rightarrow[0, \infty)$ with

$$
\int_{\delta\left(p_{0}\right)}^{\infty} \frac{d \tau}{\tau \Phi_{p_{0}}^{-1}(\tau)}=\infty
$$

for $\delta\left(p_{0}\right)>\Phi_{p_{0}}(0)$. Then $f$ is extended to a homeomorphism of $\bar{D}_{P}$ onto $\overline{D^{\prime}}{ }_{P}$.

Proof. Indeed, in the case of the hyperbolic Riemann surfaces, (9.1) and (9.2) imply (7.1) by Theorem 8.1 and, after this, Theorem 9.1 becomes a direct consequence of Theorem 7.1. In the more simple case of the elliptic and parabolic Riemann surfaces, we similarly can apply Theorem 3.1 in [28] for the Euclidean plane instead of Theorem 8.1. 
Corollary 9.1. In particular, the conclusion of Theorem 9.1 holds if

$$
\int_{D\left(p_{0}, \varepsilon_{0}\right)} e^{\alpha_{0} K_{f}(p)} d h(p)<\infty \quad \forall p_{0} \in \partial D
$$

for some $\varepsilon_{0}=\varepsilon\left(p_{0}\right)>0$ and $\alpha_{0}=\alpha\left(p_{0}\right)>0$.

Remark 9.1. Note that by Theorem 5.1 and Remark 5.1 in [16] condition (9.2) is not only sufficient but also necessary for a continuous extendibility to the boundary of all mappings $f$ with the integral restriction (9.1).

Note also that by Theorem 2.1 in [28], see also Proposition 2.3 in [27], (9.2) is equivalent to every of the conditions from the following series:

$$
\begin{gathered}
\int_{\delta\left(p_{0}\right)}^{\infty} H_{p_{0}}^{\prime}(t) \frac{d t}{t}=\infty, \quad \delta\left(p_{0}\right)>0, \\
\int_{\delta\left(p_{0}\right)}^{\infty} \frac{d H_{p_{0}}(t)}{t}=\infty, \quad \delta\left(p_{0}\right)>0, \\
\int_{\delta\left(p_{0}\right)}^{\infty} H_{p_{0}}(t) \frac{d t}{t^{2}}=\infty, \quad \delta\left(p_{0}\right)>0, \\
\int_{0\left(p_{0}\right)}^{\infty} H_{p_{0}}\left(\frac{1}{t}\right) d t=\infty, \quad \Delta\left(p_{0}\right)>0, \\
\int_{\delta_{*}\left(p_{0}\right)}^{\infty} \frac{d \eta}{H_{p_{0}}^{-1}(\eta)}=\infty, \quad \delta_{*}\left(p_{0}\right)>H_{p_{0}}(0),
\end{gathered}
$$

where

$$
H_{p_{0}}(t)=\log \Phi_{p_{0}}(t) .
$$

Here the integral in (9.5) is understood as the Lebesgue-Stieltjes integral and the integrals in (9.4) and (9.6)-(9.8) as the ordinary Lebesgue integrals.

It is necessary to give one more explanation. From the right hand sides in the conditions (9.4)-(9.8) we have in mind $+\infty$. If $\Phi_{p_{0}}(t)=0$ for $t \in\left[0, t_{*}\left(p_{0}\right)\right]$, then $H_{p_{0}}(t)=-\infty$ for $t \in\left[0, t_{*}\left(p_{0}\right)\right]$ and we complete the definition $H_{p_{0}}^{\prime}(t)=0$ for $t \in\left[0, t_{*}\left(p_{0}\right)\right]$. Note, the conditions (9.5) and (9.6) exclude that $t_{*}\left(p_{0}\right)$ belongs to the interval of integrability because 
in the contrary case the left hand sides in (9.5) and (9.6) are either equal to $-\infty$ or indeterminate. Hence we may assume in (9.4)-(9.7) that $\delta\left(p_{0}\right)>t_{0}$, correspondingly, $\Delta\left(p_{0}\right)<1 / t\left(p_{0}\right)$ where $t\left(p_{0}\right):=\sup _{\Phi_{p_{0}}(t)=0} t$, set $t\left(p_{0}\right)=0$ if $\Phi_{p_{0}}(0)>0$.

The most interesting among the above conditions is (9.6), i.e. the condition:

$$
\int_{\delta\left(p_{0}\right)}^{\infty} \log \Phi_{p_{0}}(t) \frac{d t}{t^{2}}=+\infty \quad \text { for some } \delta\left(p_{0}\right)>0
$$

Finally, it is necessary to note the restriction on nondegeneracy of boundary components of domains in Theorem 5.1 as well as in all other theorems is not essential because this simplest case is included in our previous papers [30,31].

\section{References}

[1] E. S. Afanas'eva, V. I. Ryazanov, R. R. Salimov, On mappings in Orlicz-Sobolev classes on Riemannian manifolds // Ukr. Mat. Visn., 8 (2011), No. 3, 319-342 [in Russian]; transl. in J. Math. Sci., 181 (2012), No. 1, 1-17.

[2] A. F. Beardon, The geometry of discrete groups, Graduate Texts in Math., 91, Springer-Verlag, New York, 1983.

[3] B. Bojarski, V. Gutlyanskii, O. Martio, V. Ryazanov, Infinitesimal Geometry of Quasiconformal and Bi-Lipschitz Mappings in the Plane, EMS Tracts in Mathematics, 19, Zurich EMS Publishing House, Zurich, 2013.

[4] N. Bourbaki, General topology. The main structures, Nauka, Moscow, 1968 [in Russian].

[5] C. Caratheodory, Über die Begrenzung der einfachzusammenhängender Gebiete // Math. Ann., 73 (1913), 323-370.

[6] E. F. Collingwood, A. J. Lohwator, The Theory of Cluster Sets, Cambridge Tracts in Math. and Math. Physics, 56, Cambridge Univ. Press, Cambridge, 1966.

[7] B. Fuglede, Extremal length and functional completion // Acta Math., 98 (1957), 171-219.

[8] V. Gutlyanskii, V. Ryazanov, On recent advances in boundary value problems in the plane // Ukr. Mat. Visn., 13 (2016), No. 2, 167-212; transl. in J. Math. Sci., 221 (2017), No. 5, 638-670.

[9] V. Gutlyanskii, V. Ryazanov, U. Srebro, E. Yakubov, The Beltrami Equation: A Geometric Approach, Developments in Mathematics, 26, Springer, New York etc., 2012.

[10] V. Gutlyanskii, V. Ryazanov, E. Yakubov, The Beltrami equations and prime ends // Ukr. Mat. Visn., 12 (2015), No. 1, 27-66; transl. in J. Math. Sci., 210 (2015), No. 1, 22-51. 
[11] V. Gutlyanskii, V. Ryazanov, A. Yefimushkin, On the boundary value problems for quasiconformal mappings in the plane // Ukr. Mat. Visn., 12 (2015), No. 3, 363-389; transl. in J. Math. Sci., 214 (2016), No. 2, 200-219.

[12] D. Kovtonyuk, I.Petkov, V. Ryazanov, On the Boundary Behavior of Mappings with Finite Distortion in the Plane // Lobachevskii J. Math., 38 (2017), No. 2, 290-306.

[13] D. A. Kovtonyuk, I. V. Petkov, V. I. Ryazanov, R. R. Salimov, Boundary behaviour and the Dirichlet problem for the Beltrami equations // Algebra and Analysis., 25 (2013), No. 4, 101-124 [in Russian]; transl. in St. Petersburg Math. J., 25 (2014), No. 4, 587-603.

[14] D. Kovtonyuk, I. Petkov, V. Ryazanov, R. Salimov, On the Dirichlet problem for the Beltrami equation // J. Anal. Math., 122 (2014), No. 4, 113-141.

[15] D. A. Kovtonyuk, V. I. Ryazanov, Prime ends and the Orlicz-Sobolev classes // Algebra and Analysis, 27 (2015), No. 5, 81-116 [in Russian]; transl. in St. Petersburg Math. J., 27 (2016), No. 5, 765-788.

[16] D. A. Kovtonyuk, V. I. Ryazanov, On the boundary behavior of generalized quasi-isometries // J. Anal. Math. 115 (2011), 103-119.

[17] S. L. Krushkal', B. N. Apanasov, N. A. Gusevskii, Kleinian groups and uniformization in examples and problems, Transl. of Math. Mon., 62, AMS, Providence, RI, 1986.

[18] K. Kuratowski, Topology, 1, Academic Press, New York, 1968.

[19] K. Kuratowski, Topology, 2, Academic Press, New York-London, 1968.

[20] O. Martio, V. Ryazanov, U. Srebro, E. Yakubov, Moduli in Modern Mapping Theory, Springer, New York etc., 2009.

[21] R. Näkki, Prime ends and quasiconformal mappings // J. Anal. Math. 35 (1979), $13-40$.

[22] M. H. A. Newman, Elements of the topology of plane sets of points, 2nd ed., Cambridge University Press, Cambridge, 1951.

[23] I. V. Petkov, The boundary behavior of homeomorphisms of the class $W_{\mathrm{loc}}^{1,1}$ on a plane by prime ends // Dopov. Nats. Akad. Nauk Ukr. Mat. Prirodozn. Tekh. Nauki, 2015, No. 6, 19-23 (https://doi.org/10.15407/dopovidi2015.06.019).

[24] T. Ransford, Potential Theory in the Complex Plane, Cambridge Univ. Press, Cambridge, 1995.

[25] V. Ryazanov, R. Salimov, Weakly flat spaces and bondaries in the mapping theory // Ukr. Mat. Visn., 4 (2007), No. 2, 199-234 [in Russian]; transl. in Ukrain. Math. Bull., 4 (2007), No. 2, 199-233.

[26] V. Ryazanov, R. Salimov, U. Srebro, E. Yakubov, On boundary value problems for the Beltrami equations, Complex analysis and dynamical systems V // Contemp. Math., 591 (2013), 211-242, Amer. Math. Soc., Providence, RI, 2013.

[27] V. Ryazanov, E. Sevost'yanov, Equicontinuity of mappings quasiconformal in the mean // Ann. Acad. Sci. Fenn., 36 (2011), 231-244.

[28] V. Ryazanov, U. Srebro, E. Yakubov, Integral conditions in the mapping theory // Ukr. Mat. Visn. 7 (2010), 73-87; transl. in J. Math. Sci., 173 (2011), No. 4, 397-407. 
[29] V. I. Ryazanov, S. V. Volkov, On the boundary behavior of mappings in the class $W_{\text {loc }}^{1,1}$ on Riemann surfaces // Proceedings of Inst. Appl. Math. Mech. of the NAS of Ukraine, 29 (2015), 34-53 [in Russian].

[30] V. Ryazanov, S. Volkov, On Sobolev's mappings on Riemann surfaces // ArXiv: 1604.00280v5 [math.CV] 15 Oct 2016, 24 p.

[31] V. I. Ryazanov, S. V. Volkov, On the Boundary Behavior of Mappings in the class $W_{\text {loc }}^{1,1}$ on Riemann surfaces // Complex Anal. Oper. Theory (http://dx.doi.org/10.1007/s11785-016-0618-4).

[32] V. I. Ryazanov, S. V. Volkov, On the theory of the boundary behavior of mappings in the Sobolev class on Riemann surfaces // Dopov. Nats. Akad. Nauk Ukr. Mat. Prirodozn. Tekh. Nauki, 2016, No. 10, 5-9 (http://dx.doi.org/10.15407/dopovidi2016.10.005).

[33] S. Saks, Theory of the Integral, Dover, New York, 1964.

[34] E. S. Smolovaya, Boundary behavior of ring Q-homeomorphisms in metric spaces // Ukrain. Mat. Zh., 62 (2010), No. 5, 682-689 [in Russian]; transl. in Ukrainian Math. J., 62 (2010), No. 5, 785-793.

[35] S. Stoilow, Lecons sur les principes topologiques de la theorie des fonctions analytiques, Gauthier-Villars, Paris, 1956.

[36] J. Väisälä, Lectures on n-dimensional quasiconformal mappings, Lecture Notes in Math. 229, Springer-Verlag, Berlin, 1971.

[37] G.Th. Whyburn, Analytic Topology, AMS, Providence, 1942.

\section{CONTACT INFORMATION}

Vladimir Ryazanov Institute of Applied Mathematics and Mechanics of the NAS of Ukraine, Slavyansk, Ukraine E-Mail: vl.ryazanov1@gmail.com, vlryazanov1@rambler.ru

\section{Sergei Volkov}

Institute of Applied Mathematics and Mechanics of the NAS of Ukraine, Slavyansk, Ukraine E-Mail: serhii.volkov@donntu.edu.ua 